\section{Ab Initio Study of Polonium Reveals Origins of Simple Cubic Structure}

Polonium is a rare radioactive element that has potential for a variety of engineering applications from thermoelectric power generation to dust removal and elimination of static charges. Recently, polonium has been covered in news outlets after being found in cigarettes as well as some herbal teas, and because of its nefarious use in the poisoning of Alexander Litvinenko. Beyond its notoriety, polonium is unique among all elements as being the only elemental solid found with a simple cubic crystal structure. Researchers at the Institute of Physics of Materials and Masaryk University in the Czech Republic report on ab initio calculations that explain this unique structure as a result of the Darwin and mass-velocity relativistic interactions, but not spin-orbital coupling. As an intense alpha emitter, experimental exploration of polonium is difficult, but these simulations, appearing in the July 6 issue of Physical Review Letters (016402/1-4, DOI: 10.1103/PhysRevLett. 99.016402) describe new phases in pressure regimes that should encourage work with this difficult material.

D. Legut, M. Friák, and M. Šob employ a local density approximation potential combined with relativistic effects to simulate polonium in equilibrium and stressed states. Energy profile calculations, seen in Figure 1, at increased pressures demonstrate a collapse of the energy minimum associated with simple cubic structure. These calculations express the energy as a function of the trigonal deformation path.

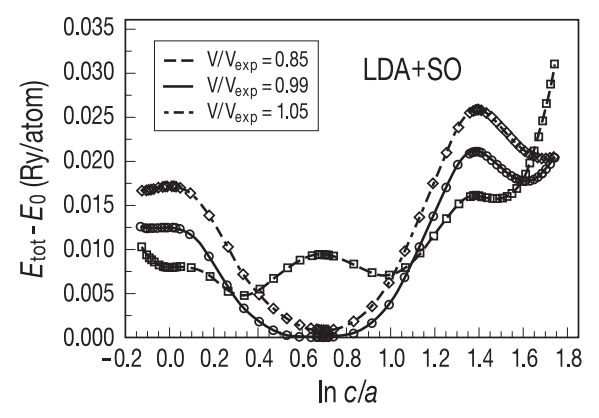

Figure 1. Local density approximation energy profiles for polonium at various stress levels. Ln c/a $=0.693$ corresponds to a simple cubic structure, for a trigonal deformation path with $c$ in the [111] direction and $a$ along a perpendicular axis. At higher pressures, the simple cubic structure destabilizes, and minima are observed corresponding to trigonal formations. Reprinted with permission from D. Legut, M. Friák, and M. Šob, Phys. Rev. Lett. 99, 016402 (2007). (c) 2007 by the American Physical Society.
A bimodal state comprised of trigonal structures, similar to the high temperature $\beta$ phase of polonium as well as the structures of isoelectronic tellurium and seleni$\mathrm{um}$, is predicted at pressures of 1-3 GPa.

The researchers then investigated the equilibrium simple cubic structure by comparing electronic structure calculations between polonium and tellurium while varying the relativistic factors active in the calculation. The spin-orbital coupling was found to not be of critical importance to achieving simple cubic structure, but the other relativistic effects were. The researchers said that the contributions from the mass-velocity term and the Darwin term, a quantum perturbation, grow larger more quickly than the spin-orbit term for larger elements in the same periodic group. These results provide a comprehensive explanation of the special combination of relativistic factors that combine to give polonium a simple cubic structure. Additionally, by predicting a bimodal phase within a narrow pressure regime, the researchers give focus to difficult experiments that may provide fruitful results.

ARTHUR FELDMAN

\section{Enzymes Can Cut or Glue Nanoparticle-Coated DNA}

Applying techniques inspired by Mother Nature, scientists can precisely construct materials at the nanometer scale. To this end, many studies have focused on using biomolecules (e.g., DNA) as templates to produce materials with specific sizes and shapes. DNA is a chain-like biopolymer with intrinsic biological functions such as self-identification, sitespecific cleavage or ligation by restriction enzymes. However, one challenge is whether DNA hybridized with nanomaterials can still be manipulated by enzymes. A group of researchers at Purdue University have found that sitespecific cleavage and ligation of DNA coated with nanoparticles can still occur.

As described in the July 24 issue of Chemistry of Materials (p. 3586; DOI: $10.1021 / \mathrm{cm} 070850 \mathrm{e}), \mathrm{A}$. Ivanisevic and co-workers demonstrated cleaving and ligation of nanoparticle-coated DNA using enzymes. The researchers were able to prepare $\mathrm{CoFe}_{2} \mathrm{O}_{4}$ nanoparticles coated with $\lambda$ phage DNA by incubating $\lambda$ phage DNA in a Tris buffer solution of $3.5 \mathrm{~nm}$ 2-pyrrolidinone-capped $\mathrm{CoFe}_{2} \mathrm{O}_{4}$ nanoparticles. Following the synthesis, they cleaved the hybrid with BamH1 and EcoR1 and then ligated the fragments with T4 ligase.

The researchers evaluated the effect of the digestion and ligation by gel electrophoresis and UV-vis spectroscopy.
Digested samples with various concentrations of nanoparticles were run on an agarose gel. The samples coated with nanoparticles produced all of the fragments expected from cleavage of the DNA sample only. This result demonstrated that the enzymes could still cut the DNA, even though it was coated with nanoparticles. The researchers also observed that the fluorescent intensity of the samples decreased with increasing nanoparticle concentration. Further experiments by UV-vis spectroscopy suggested that this effect was caused by the conformation change of the DNA. In the next step, the researchers successfully ligated the DNA fragments. Similarly to the digestion samples, the ligation samples experienced a conformation change due to the increased concentration of nanoparticles. The researchers concluded that the nanoparticle coating will not affect enzyme activity on the DNA. The researchers believe that this finding will open up methods to generate novel one-dimensional nanomaterials, which may have applications "in memory storage devices and magnetic field sensors and can provide platforms for fundamental studies of magnetic tunneling junction devices."

CHANG ZHONG

\section{Compact Polarization Rotator May Help Enable Integrated Microphotonic Systems}

The emerging class of microphotonic devices, which carry information with photons rather than electrons, may enable entire categories of novel applications from optical computers to chip-scale integrated photonic circuits. But photons have one crucial difference from electrons in standard semiconductor circuits: they are polarized, and as microphotonic devices shrink it becomes increasingly important to control this polarization at miniaturized scales. In an aticle in the August issue of Optics Letters (p. 2176), D. Beggs and T. Krauss of the University of St. Andrews, Scotland, and M. Midrio of the Universita degli Studi di Udine, Italy, report the fabrication of a new compact polarization-rotation device that should enable better polarization control in microphotonic systems.

Photon polarization is a problem in microphotonic devices because the response of small-sized waveguides built from standard silicon or III-V semiconductors to different polarizations can vary widely. To help control this, a number of passive polarization-rotation devices have been demonstrated, based either on mode evolution or birefringence. Unfortunately, devices based on mode evolution tend to 